\title{
Amygdala-hippocampal shape differences in schizophrenia: the application of 3D shape models to volumetric MR data
}

\section{Citation}

Shenton, Martha E, Guido Gerig, Robert W McCarley, Gábor Székely, and Ron Kikinis. 2002. "Amygdala-hippocampal Shape Differences in Schizophrenia: The Application of 3D Shape Models to Volumetric MR Data." Psychiatry Research: Neuroimaging 115 (1-2) (August): 15-35. doi:10.1016/s0925-4927(02)00025-2.

\section{Published Version}

doi:10.1016/s0925-4927(02)00025-2

\section{Permanent link}

http://nrs.harvard.edu/urn-3:HUL.InstRepos:28520526

\section{Terms of Use}

This article was downloaded from Harvard University's DASH repository, and is made available under the terms and conditions applicable to Other Posted Material, as set forth at http:// nrs.harvard.edu/urn-3:HUL.InstRepos:dash.current.terms-of-use\#LAA

\section{Share Your Story}

The Harvard community has made this article openly available.

Please share how this access benefits you. Submit a story.

Accessibility 


\title{
Amygdala-hippocampal shape differences in schizophrenia: the application of 3D shape models to volumetric MR data
}

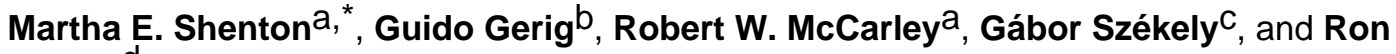 \\ Kikinis ${ }^{d}$
}

${ }^{a}$ Clinical Neuroscience Division, Laboratory of Neuroscience, Department of Psychiatry 116A, VA Boston Healthcare System, Brockton Division, 940 Belmont Street, Harvard Medical School, Brockton, MA 02301, USA bepartments of Computer Science and Psychiatry, University of North Carolina, Chapel Hill, NC, USA ${ }^{\circ}$ Communications Technology Laboratory, Swiss Federal Institute of Technology, ETH, Zurich, Switzerland dSurgical Planning Laboratory, MRI Division, Department of Radiology, Brigham and Women's Hospital, Harvard Medical School, Boston, MA, USA

\begin{abstract}
Evidence suggests that some structural brain abnormalities in schizophrenia are neurodevelopmental in origin. There is also growing evidence to suggest that shape deformations in brain structure may reflect abnormalities in neurodevelopment. While many magnetic resonance (MR) imaging studies have investigated brain area and volume measures in schizophrenia, fewer have focused on shape deformations. In this MR study we used a 3D shape representation technique, based on spherical harmonic functions, to analyze left and right amygdala-hippocampus shapes in each of 15 patients with schizophrenia and 15 healthy controls matched for age, gender, handedness and parental socioeconomic status. Left/right asymmetry was also measured for both shape and volume differences. Additionally, shape and volume measurements were combined in a composite analysis. There were no differences between groups in overall volume or shape. Left/right amygdalahippocampal asymmetry, however, was significantly larger in patients than controls for both relative volume and shape. The local brain regions responsible for the left/right asymmetry differences in patients with schizophrenia were in the tail of the hippocampus (including both the inferior aspect adjacent to parahippocampal gyrus and the superior aspect adjacent to the lateral geniculate nucleus and more anteriorly to the cerebral peduncles) and in portions of the amygdala body (including the anterior-superior aspect adjacent to the basal nucleus). Also, in patients, increased volumetric asymmetry tended to be correlated with increased left/right shape asymmetry. Furthermore, a combined analysis of volume and shape asymmetry resulted in improved differentiation between groups. Classification function analyses correctly classified $70 \%$ of cases using volume, $73.3 \%$ using shape, and $87 \%$ using combined volume and shape measures. These findings suggest that shape provides important new information toward characterizing the pathophysiology of schizophrenia, and that combining volume and shape measures provides improved group discrimination in studies investigating brain abnormalities in schizophrenia. An evaluation of shape deformations also suggests local abnormalities in the amygdala-hippocampal complex in schizophrenia.
\end{abstract}

(C) 2002 Elsevier Science Ireland Ltd. All rights reserved.

*Corresponding author. Tel.: +1-508-583-4500x2479; fax: +1-508-580-0059. martha_shenton@ @ms.harvard.edu (M.E. Shenton). 


\section{Keywords}

Schizophrenia; Magnetic resonance imaging (MRI); Amygdala-hippocampal complex; Hippocampus; Neurodevelopmental abnormalities; Shape deformations; Shape analysis; Threedimensional (3D) shape techniques; Shape descriptors

\section{Introduction}

Magnetic resonance (MR) imaging studies of schizophrenia, which began only in 1984 (Smith et al., 1984), have evolved from the use of $1-\mathrm{cm}$ slices that did not cover the whole brain to 1.5-mm slices of the entire brain (for a review, see Shenton et al., 1997, 2001; McCarley et al., 1999). This improvement in spatial resolution was needed to analyze small brain changes between normal controls and schizophrenic patients. The volume reductions observed in schizophrenia are, in fact, relatively small, on the order of 10-20\% difference from controls and, thus, improved measurement techniques were necessary before evidence could be accumulated to suggest small volume reductions in the brains of schizophrenic patients.

Such evidence has now accumulated and there has been a proliferation of MR studies documenting brain abnormalities in schizophrenia (e.g. Suddath et al., 1989, 1990; Barta et al., 1990; Bogerts et al., 1990; Dauphinais et al., 1990; DeLisi et al., 1991, 1994; Shenton et al., 1992; Andreasen et al., 1994; Marsh et al., 1994, 1997; Rossi et al., 1994a,b; Pearlson et al., 1997). In a recent review of the literature, the most robust MR findings in schizophrenia are: enlarged lateral ventricles (77\% of studies); medial temporal lobe (amygdala-hippocampal complex and/or parahippocampal gyrus) volume reduction (77\% of studies); and gray matter volume reduction of superior temporal gyrus (100\% of studies) (see Shenton et al., 1997, 2001; McCarley et al., 1999). ${ }^{1}$ There is also growing evidence to suggest that at least some structural brain abnormalities observed in schizophrenia are neurodevelopmental in origin. The general approach taken is to assume that if certain brain abnormalities could only have occurred during neurodevelopment, then an abnormal finding at a later stage of development confirms a neuro-developmental origin for that brain abnormality (e.g. Frangou and Murray, 1996; Bartley et al., 1997). For example, the sulco-gyral patterns in the brain are largely formed during the third trimester (e.g. Chi et al., 1977; Sadler, 1981; Ono et al., 1990). Thus, abnormalities in the sulco-gyral pattern of the temporal lobe in schizophrenic patients, reported in both post-mortem (e.g. Southard, 1910, 1915; Brown et al., 1986; Jakob and Beckmann, 1986) and MR studies (e.g. Kikinis et al., 1994), suggest that such alterations are the result of neurodevelopmental abnormalities. A further example of a neurodevelopmental abnormality in schizophrenia is the cavum septum pellucidi (CSP). The CSP fuses in the latter part of neural development, and therefore a space, or 'cavum', observed likely reflects deviations in neurodevelopment (e.g. Shaw and Alvord, 1969; Lewis and Mezey, 1985; Rossi et al., 1989; Sarwar, 1989; Nopoulos et al., 1996, 1997; Kwon et al., 1998). Of note, this fusion is thought to result from the rapid growth of the corpus callosum and the hippocampus, further suggesting

\footnotetext{
${ }^{1}$ Of particular note with respect to the current study, is our review of 46 MRI studies of the amygdala and hippocampus in schizophrenia (see Shenton et al., 2001), where we observed that of four studies that evaluated the amygdala alone, three showed reductions in the amygdala in patients with schizophrenia compared with control subjects. Furthermore, of 18 MRI studies that evaluated the hippocampus alone, 12 of 18 studies showed volume reduction in patients with schizophrenia compared to controls. Additionally, of seven MRI studies that evaluated the two structures separately in the same study, three out of seven showed both amygdala and hippocampal volume reduction in patients with schizophrenia compared with controls, while four out of seven studies showed no differences between patients with schizophrenia and controls for either structure. Finally, of 17 MRI studies that evaluated the amygdala-hippocampal complex, i.e. the two structures together, seven studies showed volume reduction in the amygdala-hippocampal complex in patients with schizophrenia compared with controls, while four did not; four out of 17 studies showed volume reductions in more posterior portions of the amygdalahippocampal complex in patients with schizophrenia compared with controls; and three out of 17 showed volume reductions in more anterior portions of the amygdala-hippocampal complex in patients with schizophrenia compared with controls. These findings would thus suggest that both the amygdala and the hippocampus appear to be abnormal in schizophrenia.
} 
that abnormalities in these two structures may be related, at least in part, to neurodevelopmental abnormalities (e.g. Rakic and Yakovlev, 1968). Data from our laboratory confirm an association between CSP and hippocampus; large CSP was highly correlated with reduced hippocampal volume in chronic patients (Kwon et al., 1998). (Parenthetically, the hippocampus can be affected by environmental events-see review and discussion in Gurvits et al., 1996; McEwen and Magarinos, 1997.) Additionally, planum temporale asymmetry, an important biological substrate of language, is established during neural development and it, too, has been shown to be abnormal in schizophrenia, again suggesting the importance of neurodevelopmental influences in the etiology of schizophrenia (e.g. DeLisi et al., 1994; Rossi et al., 1994a, Barta et al., 1995, 1997; Petty et al., 1995; Kwon et al., 1999).

Given the importance of neurodevelopmental influences in schizophrenia, it is of interest to note that most studies in schizophrenia have investigated area, volume and asymmetry, but fewer (e.g. Csernansky et al., 1998) have evaluated shape, which may be importantly linked to neurodevelopmental influences. For example, there is evidence to suggest that shape deformations may be associated with the physical properties of morphogenetic mechanisms that directly impact on the particular shape of brain regions during neurodevelopment (Van Essen, 1997; Van Essen and Drury, 1997; Van Essen et al., 1998). The physical tension of brain growth during neurodevelopment may lead to shape deformations that might be observed using shape measures of brain structures.

Thus, midbrain structures, likely implicated in schizophrenia which, as noted above, include both the hippocampus and corpus callosum, are important brain regions to investigate in schizophrenia as they may show shape deformations that reflect neurodevelopmental anomalies. A recent study by Thompson et al. (2000) further suggests the importance of patterns of brain growth and development, which takes place post-natally as well, and may lead to changes in volume, shape and asymmetry of brain structures in both normal and abnormal development.

In the current study, we investigated shape deformations in the amygdala-hippocampal complex in 15 male patients diagnosed with chronic schizophrenia, and 15 male controls, group matched for handedness, parental socioeconomic status and age. ${ }^{2}$ This brain region has figured prominently in many MR volume findings in schizophrenia (see reviews in Shenton et al., 1997,2001;McCarley et al., 1999), and, as noted previously, the posterior portion of the amygdala-hippocampal complex has been associated with large CSP in patients diagnosed with chronic schizophrenia (Kwon et al., 1998). This link further suggests an anomaly in neonatal development of midline brain structures. In the current study we focus on shape differences in the amygdala-hippocampal complex between groups.

Measures of shape are, nonetheless, complex. An entire field of computer science, in fact, has focused on quantitative descriptions of the shape of objects (e.g. Van Essen and Maunsell, 1980; Caviness et al., 1988; Kass et al., 1988; Bajcsy and Kovacic, 1989; Bookstein, 1989, 1997a, , , ; Evans et al., 1991; Cohen et al., 1992; Collins et al., 1992; Cootes and Taylor,

\footnotetext{
${ }^{2}$ Note: this data set was previously examined in our 1992 study (Shenton et al., 1992) where we reported a left anterior amygdalahippocampal volume reduction in patients with schizophrenia compared with control subjects. In that study, we separated the amygdala and hippocampus using the mammilary bodies. Results from our previous study showed no overall volume reduction in the combined amygdala-hippocampal complex or in the hippocampus volume between groups. Volume reduction was, however, observed in patients with schizophrenia compared with controls in the anterior portion of the amygdala-hippocampal complex, which corresponded primarily to the amygdala. In the current study, we did not separate the amygdala and hippocampus because to do so we would have had to draw an arbitrary line between the two structures, which would have resulted in a flat region on both structures. As the shape of the amygdalahippocampal complex is best appreciated as a continuous structure, we evaluated the shape of the entire amygdala-hippocampal complex, and not the amygdala and hippocampus separately. For this reason, our comparisons in this study are slightly different as they do not involve a separation of the amygdala and hippocampus, because we evaluated the amygdala-hippocampal complex (one structure) for both the volume and shape measures.
} 
1992; Brechbühler et al., 1992; Hill and Taylor, 1992; Hill et al., 1992; Talbot and Vincent, 1992; Cootes et al., 1993; Gee et al., 1993; Grenander, 1993; Christensen et al., 1994, ${ }^{1996,}$ 1997; Attali and Montanvert, 1994; Brechbühler et al., 1995; Haller et al., 1996, ${ }^{1997}$; Drury et al., 1996; Székely et al., 1996; Näf et al., 1996, ${ }^{1997}$; Bookstein, 1997a,,${ }^{\text {b c }}$; Joshi et al., 1997; Morse et al., 1998; Pizer et al., 1998; Angenent et al., 1999; Kelemen et al., 1999). Such descriptions have involved the use of a skeleton or medial axis to extract shape features (e.g. Blum, 1967, 1973; Bruce and Giblin, 1986; Talbot and Vincent, 1992; Ogniewicz, 1993; Attali and Montanvert, 1994; Kimia et al., 1995; Näf et al., 1996, 1997; August et al., 1999; Golland et al., 1999). Other approaches have included physically based shape representations such as thin-plate-splines and fiducials (e.g. Bookstein, 1989, 1997a,b,c; Pentland and Sclaroff, 1991; DeQuardo et al., 1996), surface or contour based representations (e.g. Kass et al., 1988; Brechbühler et al., 1992; Cohen et al., 1992; Cootes and Taylor, 1992; Cootes et al., 1993a, , ; Hill and Taylor, 1992; Brechbühler et al., 1995; Hill et al., 1992, 1993; Kelemen et al., 1997; Pizer et al., 1998; Angenent et al., 1999), including elastically deformable contour and surface models (e.g. Bajcsy and Kovacic, 1989; Evans et al., 1991; Collins et al., 1992; Gee et al., 1993; Christensen et al., 1994, 1996, 1997; Kelemen et al., 1999), and patternmatching methods derived from the theory of patterns by Grenander (1993) (e.g. Haller et al., 1997; Csernansky et al., 1998). A clear trend in shape analysis is toward the movement from summary measures of whole structures or objects to measures of regional differences in shape, thus incorporating more information about the properties of shape than more simple volumetric measures. Shape descriptions that are represented as high-dimensional features (Haller et al., 1996, 1997; Csernansky et al., 1998; Hogan et al., 2000; Wang et al., 2001) or features derived from a projection onto basis functions (Kelemen et al., 1999) are examples of this trend.

In this study we used an active, flexible deformable shape model to segment automatically the amygdala-hippocampal complex from MR image data. Models were trained from a set of volumes segmented manually by trained experts (derived from our previous study, Shenton et al., 1992). The surfaces of the training objects (amygdala-hippocampal complex) were then converted into parametric surface nets expanded into shape descriptions using sphericalharmonic expansion (Brechbühler et al., 1995; Székely et al., 1996). The set of shapes characterized by parameter vectors led to a statistical shape model describing the average object shape and its major modes of variation. This statistical shape model was then used for the segmentation of new datasets. Here, the average shape model was initialized based on a manual selection of three anatomical landmarks (anterior/posterior commissure and a point in the interhemispheric fissure). This shape model was driven by the object boundaries of the new image, although deformation was constrained by the statistics learned from the training sample, which significantly improves the robustness of the method (Kelemen et al., 1999). The resulting objects were represented by a set of parameters, which were then used as input for subsequent classical multivariate analyses to detect group differences in volume and in object surface descriptions (i.e. shape).

\section{Methods and materials}

\subsection{The sample}

The patient sample consisted of 15 male, chronic schizophrenics who were selected from among patients at the Brockton Veterans Affairs Medical Center. This sample has been reported in previous publications (e.g. Shenton et al., 1992). Briefly, 13 patients were hospitalized, and two were living in foster care homes. Their mean age was 37.6 years $( \pm 9.3)$, mean level of education was 11.7 years, and parental socioeconomic status (PSES) was lower middle class (3.4 \pm 0.1 , based on Hollingshead, 1965, classification). Mean age for onset of illness was 22.3 \pm 2.8 years, with mean duration of illness $15.7 \pm 8.8$ years. Patients had spent $48 \%$ of their time in the hospital since first hospitalization $(7.1 \pm 4.6$ years). Criteria for patient selection were: 
(1) DSM-III-R (American Psychiatric Association, 1987) diagnosis of schizophrenia based on information obtained from chart reviews, and from the administration of the Schedule for Affective Disorders and Schizophrenia (SADS-Lifetime Version, Spitzer and Endicott, 1978); (2) right handed; (3) between the ages 20 and 55 years old; (4) no history of electroconvulsive shock treatment; (5) no history of neurological illness; (6) no history of major alcohol/drug dependence, and no history of alcohol/drug abuse in the previous 5 years determined by DSM-III-R diagnosis; (7) no medications known to affect MR of the brain, such as steroids; and (8) verbal intelligence quotient (IQ) equal to or greater than 70, based on the Wechsler Adult Intelligence Scale-Revised (WAIS-R) information subscale (Wechsler, 1981).

The normal comparison group comprised 15 male subjects who were recruited from newspaper advertisements. These subjects were screened for neurological and psychiatric histories, and were matched to the patient sample on age, sex, handedness and PSES. Additional criteria for the comparison subjects included no history of electroconvulsant shock treatment, neurological illness, or psychiatric illness in themselves or in their first degree relatives. Additionally, the controls met the criteria of evincing no lifetime history of drug/alcohol dependence or abuse in the previous 5 years determined by DSM-III-R diagnosis.

All subjects signed informed consent prior to study participation. There were no differences between the two groups on measures such as age, weight, head circumference, PSES, or the WAISR information subscale.

\subsection{Clinical measures}

Three instruments were used to assess type and severity of symptoms: the Scale for the Assessment of Positive Symptoms (SAPS, Andreasen, 1984), the Scale for the Assessment of Negative Symptoms (SANS, Andreasen, 1981), and the Thought Disorder Index (TDI, Johnston and Holzman, 1979). Based on the Andreasen classification, 11 of the 15 patients showed predominantly positive symptoms, four showed mixed symptoms, and none showed mainly negative symptoms. The average score on the TDI was 60 , median 40 , where normal controls generally score below 5 (see Johnston and Holzman, 1979).

\subsection{Image acquisition and processing}

MR scans were obtained of the entire brain using a 1.5-T General Electric SIGNA System (GE Medical Systems, Milwaukee, WI). Two acquisition protocols were used. The first acquisition protocol consisted of 108 contiguous double echo spin-echo 3-mm axial slices, which were used to obtain total intracranial cavity contents. The imaging parameters were: Echo Time (TE) $=30$ and $80 \mathrm{~ms}$; Time to Repetition $(\mathrm{TR})=3000 \mathrm{~ms}$; field of view $=24 \mathrm{~cm}$; acquisition matrix $=256 \times 256$; and voxel dimensions $=0.9375 \times 0.9375 \times 3$ (see Shenton et al., 1992 for details).

The second acquisition protocol consisted of a 3-dimensional (3D) Fourier Transform Spoiled Gradient-Recalled (3DFT SPGR) acquisition in a steady state, resulting in 124 contiguous 1.5$\mathrm{mm}$ coronal slices, used to delineate the amygdala-hippocampal complex. The imaging parameters for this sequence were: $\mathrm{TE}=35 \mathrm{~ms}$, on repetition; nutation angle $=45^{\circ}$; field of view $=24 \mathrm{~cm}$; acquisition matrix $=256 \times 256 \times 124$; voxel dimensions $=0.9375 \times 0.9375 \times 1.5$. Automated segmentation techniques, as well as manual segmentation techniques, 3D slice editing techniques that allow reformatting in three different planes, and 3D surface rendering techniques (MRX tools developed in the Surgical Planning Laboratory, Brigham and Women's Hospital, in conjunction with General Electric), were applied to the MR datasets in order to calculate the volumes of whole brain and tissue classes, as well as to delineate the amygdalahippocampal complex. 


\subsection{Building statistical 3D shape models and automatic segmentation}

2.4.1. Amygdala-hippocampal boundary definitions-In our previous volumetric studies, we separated the amygdala and hippocampus by using an arbitrary but reliable landmark for parcellating these two structures (see Shenton et al., 1992). We acknowledged from the outset, however, that such measures were not perfect as they included both amygdala and hippocampus in slices near the mamillary bodies, the boundary that we selected to divide the two structures. For volume measures, however, we believed that because we were using the same landmarks for both patients and controls, any group differences we found would not be the result of measurement error with respect to the two structures. The individual slices were then summed to calculate the volume for the amygdala-hippocampal complex and these values were corrected for head size by using the ICC (region of interest/ICC $\times 100$ ). In the current study, we evaluated amygdala-hippocampal complex volumes from our previous study (Shenton et al., 1992; see also footnote ${ }^{2}$ ), although we did not separate the volumes of the anterior portion of the amygdala-hippocampal complex (amygdala) from the posterior portion of the amygdala-hippocampal complex (hippocampus).

For measuring shape, the separation between the amygdala and hippocampus would have meant cutting a straight line between the two structures. To place a straight line between the two structures would result in a change in the shape of the amygdala-hippocampal complex, with flat surfaces on both structures. For this reason, and because we were interested in both the amygdala and the hippocampus, we chose not to separate the two structures. Accordingly, we measured the entire amygdala-hippocampal complex in both patients and controls for both the volume and shape measures.

2.4.2. Statistical shape model-In this study we used an active, flexible deformable shape model for the automatic segmentation of the amygdala-hippocampal complex from MR image data. Here, volumetric binary segmentations of the amygdala-hippocampal complex of a training set of controls and schizophrenics were processed using a surface parametrization technique (Brechbühler et al., 1992, 1995). More specifically, object surfaces of the training objects, in this case the manually segmented amygdala-hippocampal complex from our previous study (Shenton et al., 1992), were converted into parametric surface nets and expanded into shape descriptions using spherical harmonic expansion (Brechbühler et al., 1995; Székely et al., 1996).

This surface parametrization was then expanded into a series of spherical harmonic functions, where shapes are represented as weighted sums of basis functions with varying frequencies. This technique can be best explained by referring to the well-known Fourier transform that represents signals in a frequency domain. The Fourier transform decomposes arbitrary signals into weighted sums of harmonic functions with a range of frequencies. Low frequencies represent a coarse representation of the signal in terms of waves with large wave-length, whereas high frequencies add relevant information in regard to finely detailed spikes or sharp signal changes, respectively. Similarly, in the shape description scheme presented here, low order spherical harmonic functions represent coarse features of the three-dimensional structure, whereas adding higher order functions successfully adds details of object surfaces (see Fig. 1 upper left to lower right). The weights, also called coefficients, determine the mixture of basis functions and form a compact representation to describe each object. As a result, each object is described by a set of parameters and can be reconstructed with adjustable degrees of approximation.

We chose a maximum order of 10 , resulting in $3 * 169$ coefficients per object. All the objects were aligned by translation to the surface centroid, and rotation to the three axes of the first order approximation, which is a 3D ellipsoid (Fig. 1 shows the objects reconstructed with different degrees of approximation). 
We thus began our analyses by aligning MR datasets to a standard coordinate system and by using manual segmentation from our previous study (Shenton et al., 1992). The surfaces of the segmented objects were then converted into parametric surface nets and described by spherical harmonic functions, resulting in a hierarchical 3D shape representation (Fig. 1).

The mean of the population of shapes can then be easily calculated as the mean of the vectorvalued coefficients. The large set of parameters was then reduced by applying principal component analysis (PCA). Based on the PCA, eigenvalues and eigenvectors were derived that describe the variance and eigenmodes of shape deformation, sorted by decreasing importance/ statistical significance (Cootes et al., 1994, 1995). This whole procedure results in a mean model of the amygdala-hippocampal complex and a description of its major modes of deformation. This representation can be described as a statistical object, which captures valuable information about normal and pathologic variability of organ shape.

2.4.3. Segmentation by model deformation-The statistical shape model (above) was then used for automatic segmentation of the amygdala-hippocampal complex in MR images of the 15 controls and 15 patients. A detailed description of the methodology can be found in (Székely et al., 1996; Kelemen et al., 1997, 1999). The average amygdala-hippocampus model was initialized by manually defining three landmarks in each MRI, the anterior and posterior commissure, and a third point in the hemispheric fissure. An optimization process deforms the model by attracting the model surface to the tissue boundary in the new image. Deformation of the model is, however, constrained by the shape statistics learned from the training shapes, which is a strong shape prior, and ensures a robust segmentation even in the presence of weak or non-existent boundary sections. Fig. 2 shows the initialization of the shape model (left images, blue contour and blue surface) and the resulting shapes after elastic deformation (right images, blue contour and blue surface). The result of the manual rater's segmentation is overlaid for comparison (green contour and red surface, respectively). The top row illustrates a region of interest of a sagittal cut through the three-dimensional MR image with overlay of the boundaries. The bottom row shows three-dimensional surfaces of the objects before (left) and after (right) elastic deformation (blue surfaces), with overlay of the manual expert's (i.e. manual regions of interest from the Shenton et al., 1992 study) result (red surfaces).

The automatic segmentation takes approximately 20 minutes on a standard UNIX workstation, which is a significant improvement considering the 2-3 hours of manual segmentation by an expert human rater. We tested the performance of the automatic segmentation by comparison to manual segmentations obtained by highly reliable human experts (from our previous study). The human segmentation was our 'gold standard' (albeit imperfect), and we compared this with the surface parametrization methods by testing the resulting volumes and average shape surface distance (see Fig. 3). Here, however, we faced the important question of training and testing. Given the small sample set of 15 patients with schizophrenia and 15 control subjects, we could not apply the common strategy to split the dataset into half for training and half for testing. Leave-one-out, on the other hand, had to be excluded due to computation time limitations of repeated 3D shape modeling with varying sample collections and testing on excluded subjects. As a simplified solution, we selected 21 shapes as a mixture of normal controls and patients in order to train the shape model. This model was then tested to segment the nine remaining shapes. Finally, it was used to segment all the 30 shapes using a fixed set of parameters to ensure consistency. We are aware of inherent limitations of the applied scheme, and we plan to use larger datasets in future studies.

The correlation between manual segmentation (from our previous 1992 study) and machine segmentation of the 21 left and right amygdala-hippocampal structures $(n=42)$ was $r=0.978$, $P<0.001$. Unbiased intra-class correlation was calculated as $r=0.977, P<0.001$ (Fig. 4 illustrates the correlation between manual and automatic segmentation, and shows that the 
automatic segmentation comes very close to a human-expert segmentation, but has the advantage of improved efficiency and optimal reproducibility). The result of segmentation by model deformation is not only a new object but at the same time a set of parameters representing this object. This parametric description was then used as input for subsequent shape analysis.

\subsection{Analysis of group differences}

2.5.1. Overall volume differences-We used analysis of variance measures to compare differences between groups for overall volume of the amygdala-hippocampal complex (total, and left and right). (Note: the shape measure is based on $3 * 369$ parameters, and for this reason a simple $t$-test or ANOVA cannot be computed; see below.)

2.5.2. Volume asymmetry-The volume difference index $|\mathrm{R}-\mathrm{L}| /(\mathrm{R}+\mathrm{L})$ measures the magnitude of the right-left volume difference normalized by the sum. For a statistical analysis we needed to consider that the magnitude and the ratio of the volume difference index creates non-Gaussian distributions, which might violate basic assumptions for parametric Student $t$ tests. We, therefore, used statistical techniques to deal with this problem, first by applying a monotonic transformation to the measures to create Gaussian distributions, and second by using a non-parametric method for testing the mean difference (Bradley and James, 1968).

We also used a measure more sensitive for discrimination than the $P$-values; classification rate, i.e. the percentage of correctly classified subjects after determining a discrimination function. A classification rate of 1 would indicate perfect classification, whereas 0.5 is only as good as a guess in a two-group classification. We calculated the maximum-likelihood ratio between the two groups to calculate the classification rate. Furthermore, we applied a leave-one-out analysis to determine the unbiased classification performance, which is a very important test for robustness in view of the small sample size.

2.5.3. Shape analysis-The methodology applied in this article parameterizes the surface of single objects and provides a point-by-point correspondence between homologous surface points. The shape representation by spherical harmonics is a hierarchical representation, i.e. a coarse to fine representation. We use this feature for a coarse alignment of objects prior to calculation of shape difference. Brain shapes suitable for this method (and actually shapes already studied) include: hippocampus; lateral ventricles; head of the caudate; thalamus; putamen; and globus pallidus, all simply shaped closed structures. Shapes are represented by a large set of parameters, in general several hundred, which differs from analyzing volumes represented by one scalar value. Therefore, any shape analysis requires a processing step for reducing dimensionality, e.g. principal component analysis (PCA) as used by Csernansky et al. (1998). Our method could be used similarly to reduce major shape effects to a few coefficients (major modes of shape variation) before studying group differences. This article studies asymmetry, i.e. the degree of structural difference between the left and right objects, in order to evaluate whether or not the disease process affects left and right structures to a different extent.

2.5.4. Shape asymmetry-After automatic segmentation, left and right shapes for each subject were not only segmented but also parameterized. More specifically, since the segmentation is obtained by deformation of a parametric surface model, shape parameters were automatically derived for the newly segmented objects, and expressed as a weighted sum of spherical harmonics, as explained previously.

For the shape asymmetry measures, rather than using the controls as a reference, each subject served as his own control, and deviations from overlapping the left and right amygdala- 
hippocampal surfaces were quantified for each subject in each group, and then the deviations in millimeters between the left and right surface overlap were compared between groups.

To align shapes in a coordinate system for comparison purposes, we first took advantage of the mirrored symmetry of the amygdala-hippocampal complex by mirroring the right shapes at the mid-sagittal plane, determined by manual selection of the anterior and posterior commissure and a third landmark, the interhemispheric fissure. The process of shape comparison further required a spatial alignment of objects by translation and rotation to a common coordinate system. This is illustrated in Fig. 1, upper left, which shows that the first order shape representation of all the objects is an ellipsoid with major, middle and minor axes centered at the origin of the coordinate system. These axes are used to align all the shapes with the centroid located at the origin, and the three axes of the first order ellipsoid aligned with the $x, y$ and $z$ axes. After alignment, objects can be superimposed to calculate pairwise shape differences. We chose the mean square difference (MSD) between corresponding surface points of the left and mirrored right amygdala-hippocampal shapes as a shape difference metric. Thus, the MSD provides information about the distance between overlaid left and right amygdala-hippocampal shapes measured at corresponding points. The integrated measure, however, does not provide information about whether the left or right is anomalous, only the degree to which the left and right are symmetrical.

An important question in shape analysis is not only the alignment of the object, as described above, but also the normalization of size, since objects of different size can still have similar or identical shape. The shape difference metric, described above, is sensitive to any size changes. For this reason, we scaled each object individually to normalize for size. Thus, we derived a metric that measures the residual shape difference after scaling all objects for unit size.

Finally, similar to the volume analysis, above, we determined the unbiased classification performance based on the maximum-likelihood ratio.

2.5.5. Shape differences based on combined analysis of volume and shape-In addition to the statistical analysis of left/right volume and shape asymmetry, we also tested a composite analysis of both measures. This combined analysis is motivated by the observation that volume asymmetry and shape asymmetry (after normalization for volume differences) may show significant group differences. We used a technique that is common in multivariate data analysis. Each subject is characterized by two measures, the left/right volume difference and the left/right shape difference. The two measures are then plotted in a two-dimensional joint histogram to illustrate possible correlations. Standard statistical analysis calculates first and second order statistics, here mean and covariance matrix, for each group (see quartile ellipsoids in Fig. 5, in the bottom panel) and tests the mean difference by a multivariate classification. This test assumes distributions to be approximated by parametric multidimensional Gaussian distributions. We then calculate the log likelihood ratio for each subject, which is used for standard mean difference tests and for unbiased classification performance based on leave-one-out tests.

\section{Results}

\subsection{Volume analysis}

Volumes were normalized by total intracranial volume (ICV) to control for individual head size. An ANOVA showed no differences between groups on total amygdala-hippocampal complex $(F=1.74 ;$ d.f. $=1,56 ; P=0.19)$, no differences in left or right amygdala-hippocampal complex $(F=0.74$, d.f. $=1,56, P=0.39)$, and no group by side interaction $(F=0.001$; d.f. $=$ 1,$56 ; P=0.072$ ) (see also $t$-tests in Table 1). [Note: in our earlier study (Shenton et al., 
1992), differences in volume were reported between groups for left amygdala, but this was based on a separation of the amygdala and hippocampus, and not based on the amygdalahippocampus combined, as is the case in the current study. See also Fig. 2.]

When evaluating left/right asymmetry, however, differences were statistically significant between the two groups $(F=10.40$; d.f. $=1,28 ; P<0.0032)$ (see columns labeled as 'relative volume difference' in Table 1 and plotted in Fig. 5, on the left). This finding demonstrates a larger difference between the left and right volumes in the patients compared to the controls. Finally, the unbiased classification performance was $70 \%$ ( 21 out of 30 ), which is better than chance (50\%) (see Table 2).

\subsection{Summary of volume and shape asymmetry}

In testing group asymmetry differences (see Table 1 ), we evaluated relative $R / L$ volume difference and $\mathrm{R} / \mathrm{L}$ shape difference, where we clearly show that the patient group has increased asymmetry in both measures (see also plot in Fig. 5, on the right). These measures demonstrate increased asymmetry of schizophrenia compared to control subjects.

3.2.1. Shape analysis-We calculated shape differences between groups using the mean square difference (MSD) between corresponding points of pairs of surfaces, after normalizing all shapes to unit volume. The choice of this measure was motivated by the speculation that a pathological process might result in a deformation of a structure in addition to a size change in that structure.

In order to evaluate deformation of a structure, independent of volume, we normalized shape by unit volume. Of note, results showed statistically significant group differences in the shape index $(F=5.00$; d.f. $=1,28 ; P<0.034)$. More specifically, the distance between left and right shape surfaces, expressed in millimeters, was larger in the patient than in the control group (see Fig. 5, middle), suggesting that amygdala-hippocampal shapes are less similar in schizophrenics than in controls. This result is of particular interest since we first normalized for volume differences before measuring shape asymmetry, suggesting that the shape asymmetry provides additional information to volume for studying group differences. The unbiased classification performance was $73.3 \%$ ( 22 out of 30 ), which is slightly better than the volume analysis (see Table 2).

An example of the difference between left and right amygdala-hippocampal average shapes for both controls and patients with schizophrenia can be seen in Fig. 6. Here, a graphical visualization is presented for left/right asymmetry. Shapes were uniformly scaled for normalized volumes, and the color figures display group averages of individual pairwise left/ right difference calculations. These averages were obtained by mirroring the right shapes to the left, and then overlaying the individual pairs of the amygdala-hippocampal surfaces. The signed local surface distances are mapped onto the reference shape as color, ranging from dark blue (maximum inside, the right object surface is inside the left) to red (maximum outside, the right object surface is outside the left). The green color signifies perfect overlap. A comparison between the two groups shows that the major local regions responsible for the left/right shape differences in patients with schizophrenia are in the tail of the hippocampus and in portions of the amygdala body. More specifically, deformations of the hippocampus for the patients appear to be in the inferior aspect, adjacent to the parahippocampal gyrus, and in the superior aspect, adjacent to the lateral geniculate nucleus and more anteriorly to the cerebral peduncles. Furthermore, deformations in the shape of the amygdala for the patients appear to be in the anterior-superior aspect of the amygdala, adjacent to the basal nucleus region (Peter Ratiu, M.D., expert in computational neuroanatomy and 3D rendering of neuroanatomy reviewed this figure with us). These findings suggest a major shape difference in the amygdala and in the global bending of the hippocampus in patients with schizophrenia that is not observed in 
controls. This information thus provides preliminary insight into the nature and localization of the quantitative shape asymmetry as described elsewhere in this study.

3.2.2. Combination of volume and shape features-Our volumetric analysis and the analysis of shape, normalized for individual volume, suggests that both measures should be combined for a group difference analysis. Fig. 5, on the right, shows the two-dimensional feature space with the volume index on the horizontal axis and the shape index on the vertical axis. This figure suggests that a combination of the two measures will likely improve the discrimination between patients and controls, and this is borne out using multivariate analyses, which highlight the optimal discrimination function.

More specifically, the ellipses overlaid on the data points express the quartiles of the twodimensional Gaussian distributions fitting the data. We calculated a log likelihood ratio that gives each data point a likelihood to be in either one of the two groups ( $F=11.19$; d.f. $=1,28$; $P<0.0024)$. This finding demonstrates that a combination of both features (volume and shape) results in an improved discrimination between groups. The classification performance can be determined, similar to the single variable as described above, by calculating the maximumlikelihood ratio between the two distributions. However, in view of the small sample size, and the difficulty in getting robust estimates of the two-dimensional distribution functions, we decided to apply another classifier, called support vector machine (SVM, Vapnik 1995, 1998). This classifier uses both features, the volume asymmetry index and the shape asymmetry index, to calculate the best separating discrimination function in the two-dimensional feature space as shown in Fig. 5, on the right. Again, leave-one-out was applied to get an unbiased classification performance. The unbiased classification performance, using support vector machine classification, increased to $87 \%$ ( 26 out of 30 ), which is significantly better than the classification rate obtained using either the volume or shape index alone (see above). This increase in performance is clearly visible in the right plot in Fig. 5, where the quartile ellipses give a visual impression of group separation, which can also be seen in Table 2 .

The plot in Fig. 5, on the right, suggests yet another finding. The horizontally aligned quartile ellipsoids of the control group demonstrate that left/right volume differences are smaller in controls compared to schizophrenics $(F=10.40$; d.f. $=1,28 ; P<0.0032)$. The left/right shape differences calculated from volume-normalized shapes, presented along the vertical axis, demonstrate smaller differences compared to schizophrenics $(F=5.00 ;$ d.f. $=1,28 ; P<0.034)$. The quartile ellipses visually suggest smaller variability for controls than for patients with schizophrenia.

One possible explanation for this finding is that controls have a natural left/right volume difference in the amygdala-hippocampal complex, but that their volume-normalized shapes are highly symmetric. On the other hand, patients with schizophrenia showed a much larger left/right volume lateralization but also a much larger left/right shape difference, which showed a trend for increasing linearly with increasing volume difference (correlation $r=0.31 ; P<$ $0.13)$.

\subsection{Clinical correlates of volume and shape}

We were interested in whether or not negative symptoms measured using the SANS, positive symptoms measured using the SAPS, and/or disorganized thinking measured using the TDI would be correlated with our volume or shape measures. As these were exploratory analyses, we were conservative and used two-tailed $t$-tests. Volume asymmetry was correlated $r=0.42$ ( $n=8, P=0.14)$ with total negative symptoms, $r=0.37(n=8, P=0.26)$ with total positive symptoms, and $r=0.472(n=12, P=0.058)$ with total thought disorder. Shape asymmetry was correlated $r=0.601$ with total negative symptoms $(P=0.051), r=0.098$ with total positive symptoms $(P=0.41)$, and $r=0.348(P=0.13)$ and $r-0.463(P=0.062)$ with total TDI and 
total TDIlog, respectively. A combined shape and volume measure correlated $r=0.366(P=$ $0.18)$ with total negative symptoms, $r=0.393(P=0.16)$ with total positive symptoms, and $r$ $=0.476(P=0.060)$ with total TDI. As the sample sizes here are quite small, albeit with moderate effect sizes for total negative symptoms and TDI (i.e., 0.4 or more), confirmation of these findings in a new sample is needed.

\section{Discussion}

We evaluated volume and shape differences in the amygdala-hippocampal complex between patients diagnosed with schizophrenia and normal comparison subjects. We found no differences in overall amygdala-hippocampal volume between groups, but we did report both volume and shape asymmetry differences, which were significantly larger in the patient than in the control group. More specifically, we observed a closer correspondence between the shapes of the left and right amygdala-hippocampal complex in normal controls than we observed in patients with schizophrenia. In the patient group, there was a greater mean square difference (MSD) between corresponding points of pairs of surfaces, after normalizing all shapes to unit volume, between the left and right amygdala-hippocampal shapes than was observed for the control group.

Findings of laterality differences in schizophrenia in the amygdala-hippocampal complex have been reported by other groups evaluating volume (see discussion and citations in Section 1 and see also the footnotes), and thus our finding is consistent with what has been reported in the literature. Our findings of shape differences in the amygdala-hippocampal complex in schizophrenic patients are also consistent with the findings reported for the hippocampus by Csernansky et al. (1998), although these investigators reported deformations in the hippocampal head on both the left and the right sides in schizophrenic patients. These findings indicate that morphology reflects a pathological process. The measures presented in this article, however, do not fully explain the nature and the precise location and lateralization of this effect, although there is preliminary evidence to suggest that the tail of the hippocampus and portions of the amygdala body are abnormal in schizophrenia.

The left/right asymmetry findings in this study can be compared to recent findings by Wang et al. (2001), with the difference being that we studied the amygdala-hippocampal complex rather than the hippocampus alone. In comparison to shape analysis by high-dimensional warping (Csernansky et al., 1998; Wang et al., 2001; Hogan et al., 2000; Joshi et al., 1997), we used a completely different methodology that extracts and parameterizes individual objects, determines spatial alignment and normalization, and then calculates a shape difference metric (mean square distance between corresponding surfaces) to express shape difference. A new component is the individual normalization by volume before calculation of the shape difference. We observed that most shape difference metrics can be sensitive to volume changes, which means that even identical shapes with different volumes would present a shape difference. In the application described herein, left/right volume differences have been found as one discriminating feature. As a logical consequence, we decided to calculate shape differences that were 'orthogonal' to volume.

The shape lateralization in our study shows more lateralization for schizophrenics than controls. The result is significant at less than the 5\% level ( $F=5.00$; d.f. $=1,28 ; P<0.034)$. This finding, in itself, suggests that the shape of this structure is altered in patients diagnosed with schizophrenia. A significant improvement in the separation between groups was demonstrated by combining both volume and shape features, resulting in an increase of the classification performance from 70 to 73 to $87 \%$. This result corroborates the fact that shape information in addition to volume analysis improves group discrimination and potentially helps to explain pathology. The combined measure turns out to be a more sensitive and critical 
measure of neural abnormalities, which are not fully captured by volume or by shape measures alone.

Correlations of volume and shape asymmetry showed moderate correlations for total negative symptom scores and for total thought disorder, suggesting that anomalies in amygdalahippocampal asymmetry may be correlated with both negative symptoms and disorganized thinking. Though intriguing, the sample size is small, and the analyses were exploratory in nature, thus necessitating confirmation in a new sample.

The overlaid average left and mirrored right amygdala-hippocampal shapes for each group are shown in Fig. 6. This overlap suggests a shape difference in the amygdala and in the global bending of the hippocampus in patients with schizophrenia that is not observed in controls. Future studies will likely provide an intuitive shape description using natural language terms (length, bending, local width, local curvature) in order to explain shape and shape changes in a more anatomically meaningful way.

In summary, the development of automated measures of shape for brain structures is important as it may lead to a deeper understanding of the role of neurodevelopment in the pathophysiology of schizophrenia. Such an understanding may also further our appreciation of static vs. progressive brain changes in brain disorders such as schizophrenia and may lead to more informed pharmacological treatment. Studies that focus on delineating more local regions of shape difference and automated measures of both shape and volume using template-driven automated segmentation techniques will enable us to evaluate a large number of brain regions in a large population of patients.

\section{Acknowledgments}

This research was supported in part by funds from the National Institute of Mental Health, including grants NIMH K02 MH-01110 and R01 MH-50747 (Dr Shenton), and NIMH R01-40977 (Dr McCarley); by VA MERIT Awards from the Department of Veterans Affairs (Drs McCarley and Shenton), by the Medical Research Service and Brockton VA Schizophrenia Center of the Department of Veteran Affairs (Dr McCarley); and by P01 CA67165, P01 AG04953, R01 RR11747 and P41 RR13218 (Dr Kikinis). We would also like to thank Peter Ratiu, M.D., an expert in computational neuroanatomy and 3D renderings of anatomy. His assistance was invaluable in describing the neuroanatomical regions of the amygdala-hippocampal complex that showed shape deformations between the left and right amygdala-hippocampal complex in patients with schizophrenia.

\section{References}

American Psychiatric Association on Nomenclature and Statistics. Diagnostic and Statistical Manual of Mental Disorders, revised. 3rd edition. Washington, DC: American Psychiatric Association; 1987.

Andreasen, NC. Scale for the Assessment of Negative Symptoms (SANS). Iowa City: University of Iowa College of Medicine; 1981.

Andreasen, NC. Scale for the Assessment of Positive Symptoms (SAPS). Iowa City: University of Iowa College of Medicine; 1984.

Andreasen NC, Flashman L, Flaum M, Arndt S, Swayze V II, O'Leary DS, Ehrhardt JC, Yuh WTC. Regional brain abnormalities in schizophrenia measured with magnetic resonance imaging. JAMA 1994;272:1763-1769. [PubMed: 7966925]

Angenent S, Haker S, Tannenbaum A, Kikinis R. On the Laplace-Beltrami operator and brain surface flattening. IEEE Transactions on Medical Imaging 1999;18:700-711. [PubMed: 10534052]

Attali D, Montanvert A. Semicontinuous skeletons of 2D and 3D shapes. Proceedings from the Second International Workshop on Visual Form 1994:32-41.

August, J.; Tannebaum, A.; Zucker, S. On the evolution of the skeleton; Proceedings of ICCV, Corfu; Greece. 1999. p. 315-322.

Bajcsy, R.; Kovacic, S. Mutiresolution elastic matching. Proceedings of the Conference on Computer Vision; Graphics and Imaging; 1989. p. 1-21. 
Barta PE, Pearlson GD, Brill LB, Royall R, McGilchrist IK, Pulver AE, Powers RE, Casanova MF, Tien AY, Frangou S, Petty PG. Planum temporale asymmetry reversal in schizophrenia: replication and relationship to gray matter abnormalities. American Journal of Psychiatry 1997;154:661-667.

[PubMed: 9137122]

Barta PE, Pearlson GD, Powers RE, Richards SS, Tune LE. Auditory hallucinations and smaller superior temporal gyrus volume in schizophrenia. American Journal of Psychiatry 1990;147:1457-1462. [PubMed: 2221156]

Barta PE, Petty RG, McGilchrist I, Lewis RW, Jerram M, Casanova MF, Powers RE, Brill LB II, Pearlson GD. Asymmetry of the planum temporale: methodological considerations and clinical associations. Psychiatry Research: Neuroimaging 1995;61:137-150.

Bartley AJ, Jones DW, Weinberger DR. Genetic variability of human brain size and cortical gyral patterns. Brain 1997;120:257-269. [PubMed: 9117373]

Blum, H. A transformation for extracting new descriptors of shape. In: Walthen-Dunn, W., editor. Models for the Perception of Speech and Visual Form. Cambridge, MA: MIT Press; 1967.

Blum H. Biological shape and visual science. Journal of Theoretical Biology 1973;38:205-287. [PubMed: 4689997]

Bogerts B, Ashtari M, Degreef G, Alvir JM, Bilder RM, Lieberman JA. Reduced temporal limbic structure volumes on magnetic resonance images in first episode schizophrenia. Psychiatry Research: Neuroimaging 1990;35:1-13.

Bookstein FL. Principal warps: thin-plate splines and the decomposition of deformations. IEEE PAMI 1989;11:567-585.

Bookstein FL. Shape and information in medical images: a decade of the morphometric synthesis. Computer Vision and Image Understanding 1997a;66:97-118.

Bookstein FL. Landmark methods for forms without landmarks: localizing group differences in outline shape. Medical Image Analysis 1997b;1:225-243. [PubMed: 9873908]

Bookstein, FL. Quadratic variation of deformation. In: Duncan, J.; Gindi, G., editors. Proceedings IPMI. Vol. 1230. Springer LNCS; 1997c. p. 15-28.

Bradley, JV. Distribution Free Statistical Tests. Englewood Cliffs, NJ: Prentice Hall; 1968.

Brechbühler C, Gerig G, Kübler O. Surface parametrization and shape description. Visualization in Biomedical Computing 1992:80-89.

Brechbühler C, Gerig G, Kübler O. Parametrization of closed surfaces for 3-D shape description. CVGIP: Image Understanding 1995;61:154-170.

Brown R, Colter N, Corsellis JAN, Crow TJ, Frith CD, Jagoe R, Johnstone EC, Marsh L. Postmortem evidence of structural changes in schizophrenia: differences in brain weight, temporal horn area and parahippocampal gyrus compared with affective disorders. Archives of General Psychiatry 1986;43:36-42. [PubMed: 2935114]

Bruce J, Giblin B. Growth, motion and one-parameter families of asymmetry sets. Proceedings of the Royal Society of Edinburgh 1986;104A:179-204.

Caviness VS, Filipek PA, Kennedy DN. Magnetic resonance technology in human brain science: blueprint for a program based upon morphometry. Brain and Development 1988;11:1-13. [PubMed: 2646959]

Chi JG, Dooling EC, Gilles FH. Gyral development of the brain. Annals of Neurology 1977;1:86-93. [PubMed: 560818]

Christensen, GE.; Miller, MI.; Vannier, M. Proceedings of an AAAI Workshop: Application of Computer Vision in Medical Image Processing. Stanford University: 1994. A 3D deformable magnetic resonance textbook based on elasticity; p. 153-156.

Christensen GE, Rabbit RD, Miller MI. Deformable templates using large deformation kinematics. IEEE Transactions on Image Processing 1996;5:1435-1447. [PubMed: 18290061]

Christensen GE, Joshi SC, Miller MI. Volumetric transformation of brain anatomy. IEEE Transactions on Medical Imaging 1997;16:864-877. [PubMed: 9533586]

Cohen I, Cohen LD, Ayache N. Using deformable surfaces to segment 3-D images and infer differential structures. CVGIP: Image Understanding 1992;56:242-263. 
Collins DL, Peters TM, Dai W, Evans AC. Model based segmentation of individual brain structures from MRI data. SPIE Visualization in Biomedical Computing 1992;1801:10-23.

Cootes TF, Cooper D, Taylor CJ, Graham J. Active shape models-their training and application. Computer Vision and Image Understanding 1995;61:31-59.

Cootes T, Hill A, Taylor C, Haslam J. The use of active shape models for locating structures in medical images. IPMI, Flagstaff, AZ 1993a:3-47.

Cootes, TF.; Hill, A.; Taylor, CJ.; Haslam, J. The use of active shape models for locating structures in medical images. Information Processing in Medical Imaging. In: Barrett, HH.; Gmitro, AF., editors. Lecture Notes in Computer Science. Vol. 687. Berlin: Springer-Verlag; 1993b. p. 33-47.

Cootes TF, Hill A, Taylor CJ, Haslam J. The use of active shape models for locating structures in medical images. Image and Vision Computing 1994;12:355-366.

Cootes, T.; Taylor, C. Active shape models—-'smart snakes'; British Mach Vision Conf; Berlin: SpringerVerlag; 1992. p. 266-275.

Csernansky JC, Joshi SC, Wang L, Gado M, Miller JP, Grenander U, Miller MI. Hippocampal morphometry in schizophrenia by high dimensional brain mapping. Proceedings of the National Academy of Sciences of the Unites States of America 1998;95:11406-11411.

Dauphinais DI, DeLisi LE, Crow TJ, Alexandropoulos K, Colter N, Tuma I, Gershon ES. Reduction in temporal lobe size in siblings with schizophrenia: a magnetic resonance imaging study. Psychiatry Research: Neuroimaging 1990;35:137-147.

DeLisi LE, Hoff AL, Neale C, Kushner M. Asymmetries in the superior temporal lobe in male and female first-episode schizophrenic patients: measures of planum temporale and superior temporal gyrus by MRI. Schizophrenia Research 1994;12:19-28. [PubMed: 8018582]

DeLisi LE, Hoff AL, Schwartz JE, Shields GW, Halthore SN, Gupta SM, Henn FA, Anand AK. Brain morphology in first-episode schizophrenic-like psychotic patients: a quantitative magnetic resonance imaging study. Biological Psychiatry 1991;29:159-175. [PubMed: 1995085]

DeQuardo JR, Tandon R, Brunberg JA, Green WD, Bookstein FL. Spatial relationships of neuroanatomic landmarks in schizophrenia. Psychiatry Research: Neuroimaging 1996;67:81-95.

Drury HA, Van Essen DC, Anderson CH, Lee CW, Coogan TA, Lewis JW. Computerized mappings of the cerebral cortex. A multiresolution flattening method and a surface-based coordinate system. Journal of Cognitive Neuroscience 1996;8:1-28. [PubMed: 11539144]

Evans A, Dai W, Collins L, Neelin P, Marret S. Warping of a computerized 3D atlas to match brain image volumes for quantitative neuroanatomical and functional analysis. Image Processing 1991;1445:236246.

Frangou S, Murray RM. Imaging as a tool in exploring the neurodevelopment and genetics of schizophrenia. British Medical Bulletin 1996;52:587-596. [PubMed: 8949259]

Gee JC, Reivich M, Bajcsy R. Elastically deforming 3D atlas to match anatomical brain images. Journal of Computer Assisted Tomography 1993;17:225-236. [PubMed: 8454749]

Golland, P.; Grimson, WE.; Kikinis, R. Statistical shape analysis using fixed topology skeletons: corpus callosum study. In: Kuba, A., editor. IPMI LNCS. Vol. 161. Berlin: Springer-Verlag; 1999. p. 382-387.

Grenander, U. General Pattern Theory. London, England: Oxford University Press; 1993.

Gurvits TV, Shenton ME, Hokama H, Ohta H, Lasko NB, Gilbertson MW, Orr SP, Kikinis R, Jolesz FA, McCarley RW, Pitman RK. Magnetic resonance imaging study of hippocampal volume in chronic, combat related posttraumatic stress disorder. Biological Psychiatry 1996;40:1091-1099. [PubMed: 8931911]

Haller JW, Banerjee A, Christensen GE, Gado M, Joshi S, Miller MI, Sheline YI, Vannier MW, Csernansky JG. 3D hippocampal morphometry by high dimensional transformation of a neuroanatomical atlas. Radiology 1997;202:504-510. [PubMed: 9015081]

Haller J, Christensen GE, Joshi SC, Newcomer JW, Miller MI, Csernansky JG, Vannier MW. Hippocampal MR imaging morphometry by means of general pattern matching. Radiology 1996;199:787-791. [PubMed: 8638006]

Hill, A.; Cootes, TF.; Taylor, CJ. A generic system for image interpretation using flexible templates; Proc. British Mach. Vision Conf; Springer; 1992. p. 276-285. 
Hill A, Taylor CJ. Model-based image interpretation using genetic algorithms. Image and Vision Computing 1992;10:295-300.

Hill, A.; Thornham, A.; Taylor, CJ. Model-based interpretations of 3-D medical images; Proc. British Mach. Vision Conf; BMVC Press; 1993. p. 239-348.

Hogan RE, Mark KE, Wang L, Joshi S, Miller MI, Bucholz RD. Mesial temporal sclerosis and temporal lobe epilepsy: MR imaging deformation-based segmentation of the hippocampus in five patients. Radiology 2000;216:291-297. [PubMed: 10887264]

Hollingshead, AB. Two-Factor Index of Social Position. New Haven, CT: Yale University Press; 1965.

Jakob H, Beckmann H. Prenatal developmental disturbances in the limbic allocortex in schizophrenics. Journal of Neural Transmission 1986;65:303-326. [PubMed: 3711886]

Johnston, MH.; Holzman, PS. Assessing Schizophrenic Thinking: A Clinical and Research Instrument for Measuring Thought Disorder. San Francisco, CA: Jossey-Bass; 1979.

Joshi S, Grenander U, Miller MI. On the geometry and shape of brain sub-manifolds. International Journal of Pattern Recognition and Artificial Intelligence 1997;11:1317-1343.

Kass M, Witkin A, Terzopoulos D. Snakes: active contour models. International Journal Computer Vision 1988;1:321-331.

Kelemen, A.; Székely, G.; Gerig, G. MB3IA Workshop, ICCV98, Bombay, India. TR-178 Technical Report. ETH, Zurich: Image Science Lab; 1997. Three-dimensional model-based segmentation.

Kelemen A, Székely G, Gerig G. Elastic model-based segmentation of 3D neuroradiological data sets. IEEE Transactions on Medical Imaging 1999;18:828-839. [PubMed: 10628943]

Kikinis R, Shenton ME, Gerig G, Hokama H, Haimson J, O’Donnell BF, Wible CG, McCarley RW, Jolesz FA. Temporal lobe sulco-gyral pattern anomalies in schizophrenia: an in vivo MR threedimensional surface rendering study. Neuroscience Letters 1994;183:7-12. [PubMed: 7891892]

Kimia B, Tannebaum A, Zucker S. Shapes, shocks, and deformations: I: The components of shape and the reaction-diffusion space. International Journal of Computer Vision 1995;15:189-224.

Kwon JS, McCarley RW, Anderson JE, Hirayasu Y, Fischer IA, Kikinis R, Jolesz FA, Shenton ME. Left planum temporale volume reduction in schizophrenia. Archives of General Psychiatry 1999;56:142148. [PubMed: 10025438]

Kwon JS, Shenton ME, Hirayasu Y, Salisbury DF, Fischer IA, Dickey CC, Yurgelun-Todd D, Tohen M, Kikinis R, Jolesz FA, McCarley RW. MRI study of cavum septi pellucidi in schizophrenia, affective disorder and schizotypal personality disorder. American Journal of Psychiatry 1998;155:509-515. [PubMed: 9545997]

Lewis SW, Mezey GC. Clinical correlates of septum pellucidum cavities: an unusual association in psychosis. Psychological Medicine 1985;15:43-54. [PubMed: 2581281]

Marsh L, Harris D, Lim KO, Beal M, Hoff AL, Minn K, Csernansky JG, DeMent S, Faustman WO, Sullivan EV, Pfefferbaum A. Structural magnetic resonance imaging abnormalities in men with severe chronic schizophrenia and an early age at clinical onset. Archives of General Psychiatry 1997;54:1104-1112. [PubMed: 9400346]

Marsh L, Suddath RL, Higgins N, Weinberger DR. Medial temporal lobe structures in schizophrenia: relationship of size to duration of illness. Schizophrenia Research 1994;11:225-238. [PubMed: 8193061]

McCarley RW, Wible CG, Frumin M, Hirayasu Y, Levitt JJ, Fischer IA, Shenton ME. MRI anatomy of schizophrenia. Biological Psychiatry 1999;45:1099-1119. [PubMed: 10331102]

McEwen BS, Magarinos AM. Stress effects on morphology and function of the hippocampus. Annals of the New York Academy of Sciences 1997;821:271-284. [PubMed: 9238211]

Morse BS, Pizer SM, Puff D, Gu C. Zoom-invariant vision of figural shape: effects on cores of image disturbances. Computer Vision and Image Understanding 1998;69:72-86.

Näf M, Kübler O, Kikinis R, Shenton ME, Székeley G. Characterization and recognition of 3D organ shapes in medical image analysis using skeletonization. IEEE Mathematical Methods in Biomedical Image Analysis 1996;1:139-150.

Näf M, Székeley G, Kikinis R, Shenton ME, Kübler O. 3D voronoi skeletons and their usage for the characterization and recognition of 3D organ shape. Computer Vision and Image Understanding 1997;66:640-646. 
Nopoulos P, Swayze V, Andreasen NC. Pattern of brain morphology in patients with schizophrenia and large cavum septi pellucidi. Journal Neuropsychiatry and Clinical Neurosciences 1996;8:147-152.

Nopoulos P, Swayze V, Flaum M, Erhardt JC, Yuh WT, Andreasen NC. Cavum septi pellucidi and patients with schizophrenia as detected by magnetic resonance imaging. Biological Psychiatry 1997;41:1102-1108. [PubMed: 9146821]

Ogniewicz, RL. Discrete Voronoi Skeletons. Konstanz, Zurich: Hartung-Goree Verlag; 1993.

Ono, M.; Kubik, S.; Abernathy, CD. Atlas of the Cerebral Sulci. New York: Thieme Medical Publishers; 1990.

Pearlson GD, Barta PE, Powers RE, Menon RR, Richards SS, Aylward EH, Federman EB, Chase GA, Petty RG, Tien AY. Medial and superior temporal gyrus volumes and cerebral asymmetry in schizophrenia versus bipolar disorder. Biological Psychiatry 1997;41:1-14. [PubMed: 8988790]

Pentland A, Sclaroff AP. Closed-form solutions for physically based shape modelling and recognition. IEEE PAMI 1991;13:715-729.

Petty RG, Barta PE, Pearlson GD, McGilchrist IK, Lewis RW, Tien AY, Pulver A, Vaughn DD, Casanova MF, Powers RE. Reversal of asymmetry of the planum temporale in schizophrenia. American Journal of Psychiatry 1995;152:715-721. [PubMed: 7726311]

Pizer SM, Eberly D, Fritsch DS, Morse BS. Zoom-invariant vision of figural shape: the mathematics of cores. Computer Vision and Image Understanding 1998;69:55-71.

Rakic P, Yakovlev PI. Development of corpus callosum and cavum septi in man. Journal of Comparative Neurology 1968;132:355-362.

Rossi A, Stratta P, Gallucci M, Passariello R, Casacchia M. Quantification of corpus callosum and ventricles in schizophrenia with nuclear magnetic resonance imaging: a pilot study. American Journal of Psychiatry 1989;146:99-101. [PubMed: 2912255]

Rossi A, Serio A, Stratta P, Petruzzi C, Schiazza G, Mancini F, Casacchia M. Planum temporale asymmetry and thought disorder in schizophrenia. Schizophrenia Research 1994a;12:1-7. [PubMed: 8018581]

Rossi A, Stratta P, Mancini F, Gallucci M, Mattei P, Core I, Di Michele V, Casacchia M. Magnetic resonance imaging findings of amygdala-anterior hippocampus shrinkage in male patients with schizophrenia. Psychiatry Research: Neuroimaging 1994b;52:43-53.

Sadler, TW. Langman's Medical Embryology. Baltimore: Williams \& Wilkins; 1981.

Sarwar M. The septum pellucidum: normal and abnormal. American Journal of Neuroradiology 1989;10:989-1005. [PubMed: 2505543]

Shaw CM, Alvord CE. Cavum septi pellucidi et vergae: their normal and pathological state. Brain 1969;92:213-224. [PubMed: 5774029]

Shenton ME, Dickey CC, Frumin M, McCarley RW. A review of MRI findings in schizophrenia. Schizophrenia Research 2001;49:1-52. [PubMed: 11343862]

Shenton, ME.; Wible, CG.; McCarley, RW. Brain Imaging in Clinical Psychiatry. New York: Marcel Dekker; 1997. A review of magnetic resonance imaging studies of brain abnormalities in schizophrenia; p. 297-380.

Shenton ME, Kikinis R, Jolesz FA, Pollak SD, LeMay M, Wible CG, Hokama H, Martin J, Metcalf D, Coleman M, McCarley RW. Abnormalities of the left temporal lobe and thought disorder in schizophrenia: a quantitative magnetic resonance imaging study. New England Journal of Medicine 1992;327:604-612. [PubMed: 1640954]

Smith RC, Calderon M, Ravichandran GK, Largen J, Vroulis G, Shvartsburd A, Gordon J, Schoolar JC. Nuclear magnetic resonance in schizophrenia: a preliminary study. Psychiatry Research 1984;12:137-147. [PubMed: 6591219]

Southard EE. A study of the dementia praecox group in the light of certain cases showing anomalies or scleroses in particular brain regions. American Journal of Insanity 1910;67:119-176.

Southard EE. On the topographic distribution of cortex lesions and anomalies in dementia praecox with some account of their functional significance. American Journal of Insanity 1915;71:603-671.

Spitzer, RL.; Endicott, J. Schedule for Affective Disorders and Schizophrenia-Lifetime Version. 3rd edition. New York: New York State Psychiatric Institute, Biometrics Research; 1978. 
Suddath RL, Casanova MF, Goldberg TE, Daniel DG, Kelsoe JR, Weinberger DR. Temporal lobe pathology in schizophrenia: a quantitative magnetic resonance imaging study. American Journal of Psychiatry 1989;146:464-472. [PubMed: 2929746]

Suddath RL, Christison GW, Torrey EF, Casanova MF, Weinberger DR. Anatomical abnormalities in the brains of monozygotic twins discordant for schizophrenia. New England Journal of Medicine 1990;332:789-794. [PubMed: 2308615]

Székely G, Kelemen A, Brechbühler C, Gerig G. Segmentation of 2-D and 3-D objects from MRI volume data using constrained elastic deformations of flexible Fourier contour and surface models. Medical Image Analysis 1996;1:19-34. [PubMed: 9873919]

Talbot, H.; Vincent, L. Euclidean skeletons and conditional bisectors; Proceedings SPIE Conf. Medical Imaging V: Image Processing; 1992. p. 862-876.

Thompson PM, Giedd JN, Woods RP, MacDonald D, Evans AC, Toga AW. Growth patterns in the developing human brain detected using continuum-mechanical tensor mapping. Nature 2000;404:190-193. [PubMed: 10724172]

Van Essen DC. A tension-based theory of morphogenesis and compact wiring in the central nervous system. Nature 1997;385:313-318. [PubMed: 9002514]

Van Essen DC, Drury HA. Structural and functional analyses of human cerebral cortex using a surfacebased atlas. Journal of Neuroscience 1997;17:7079-7102. [PubMed: 9278543]

Van Essen DC, Drury HA, Joshi S, Miller MI. Functional and structural mapping of human cerebral cortex: solutions are in the surfaces. Proceedings of the National Academy of Sciences of the United States of America 1998;95:788-795. [PubMed: 9448242]

Van Essen DC, Maunsell JHR. Two-dimensional maps of the cerebral cortex. Journal of Comparative Neurology 1980;191:255-281. [PubMed: 7410593]

Vapnik, VN. The Nature of Statistical Learning Theory. Springer Verlag; 1995.

Vapnik, VN. Statistical Learning Theory. Springer Verlag; 1998.

Wang L, Joshi SC, Miller MI, Csernansky JG. Statistical analysis of hippocampal asymmetry in schizophrenia. Neuroimage 2001;14:531-545. [PubMed: 11506528]

Wechsler, D. Wechsler Adult Intelligence Scale-Revised. New York: Harcourt-Brace-Jovanovich Inc; 1981. 


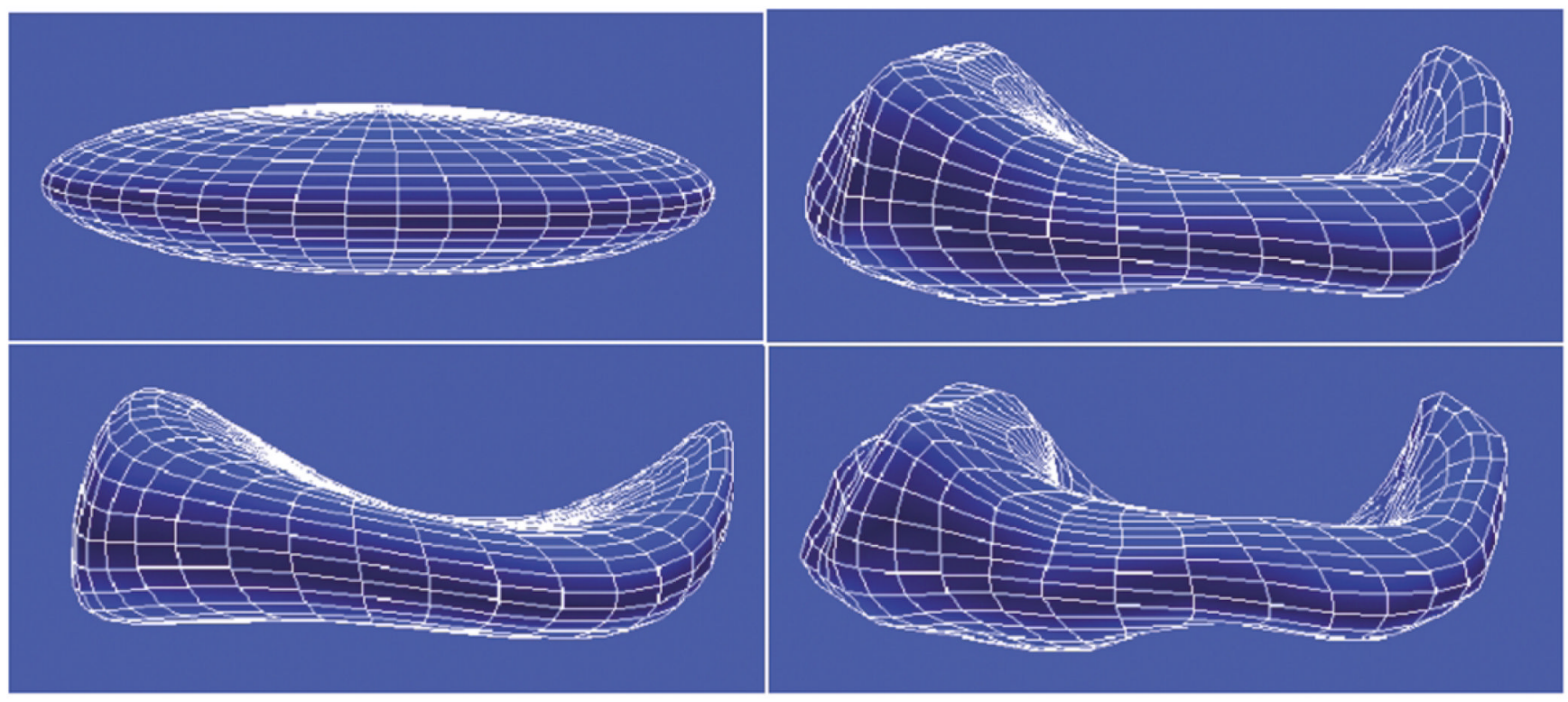

Fig. 1.

Hierarchical Fourier surface representation of the amygdala-hippocampal complex. This figure shows reconstructions up to order 1 (top left), 3 (bottom left), 7 (top right) and 12 (bottom right). Of note, more and more details are added with increasing order (i.e., from 1 to 12). The first order representation is an ellipsoid and is used for a spatial alignment of shapes by translation and rotation. 


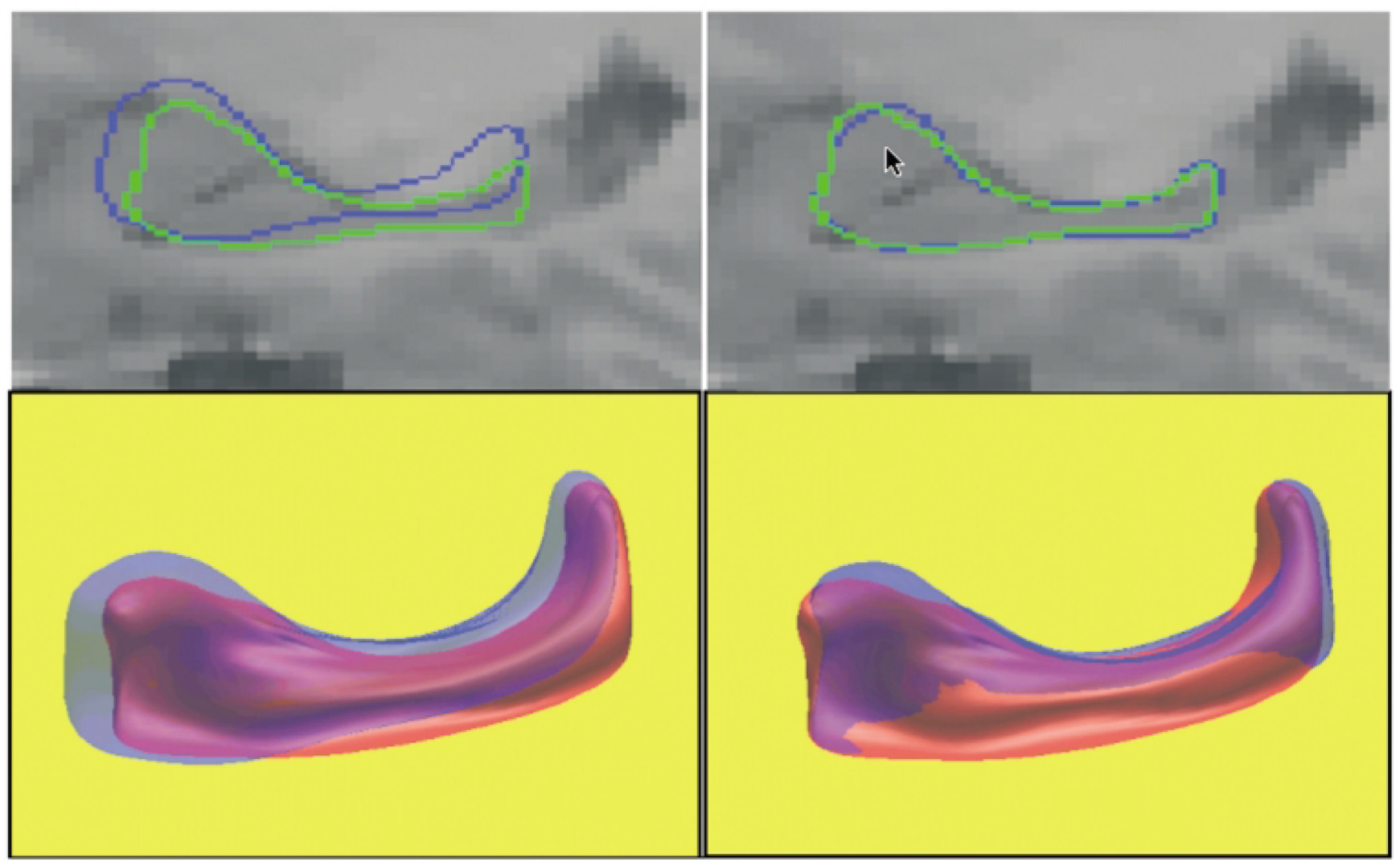

Fig. 2.

Automatic segmentation of 3D amygdala-hippocampal complex using surface-based modeldeformation (3D Fourier snake). Figures in the left panel show the 3D initialization based on Talairach coordinates (blue contour, blue surface), and a manual rater's segmentation (green contour, blue surface). Figures in the right panel show the resulting segmentation after 3D model deformation. 


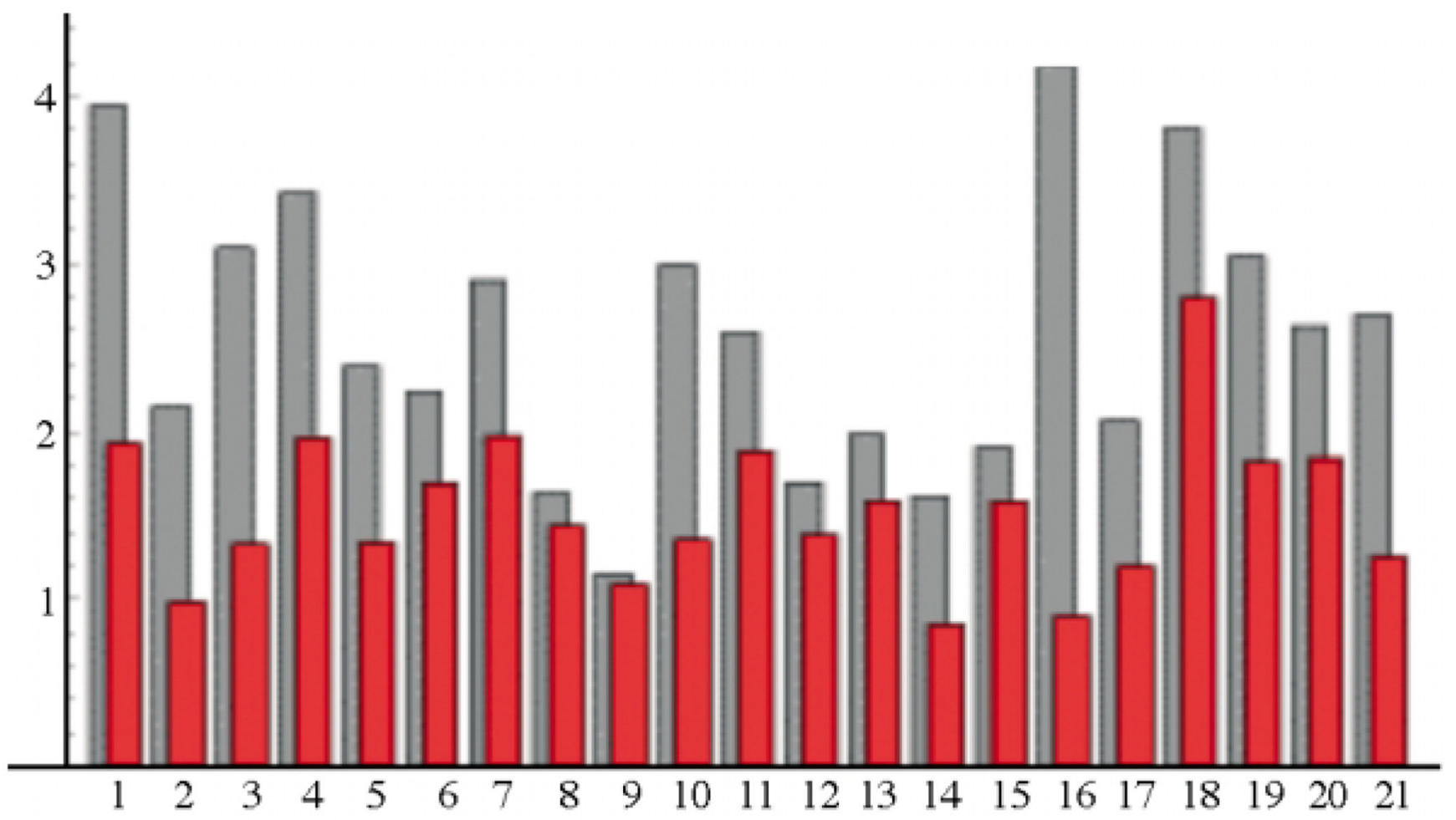

Fig. 3.

Shape distance measures are displayed for the segmentations of the left amygdala-hippocampal complex for 21 individuals. The plot illustrates quantitative evaluation of the shape differences for the manual slice-by-slice segmentation and the automated segmentation. The horizontal axis displays the 21 individual cases, and the vertical measurement displays the square root of the mean square distance in millimeters between the surfaces of the 3D object pairs. The light gray bars represent the shape distance at model initialization, and the dark red bars represent the shape distance after model-based segmentation by elastic model deformation. 


\section{Manual vs. automatic segmentation}

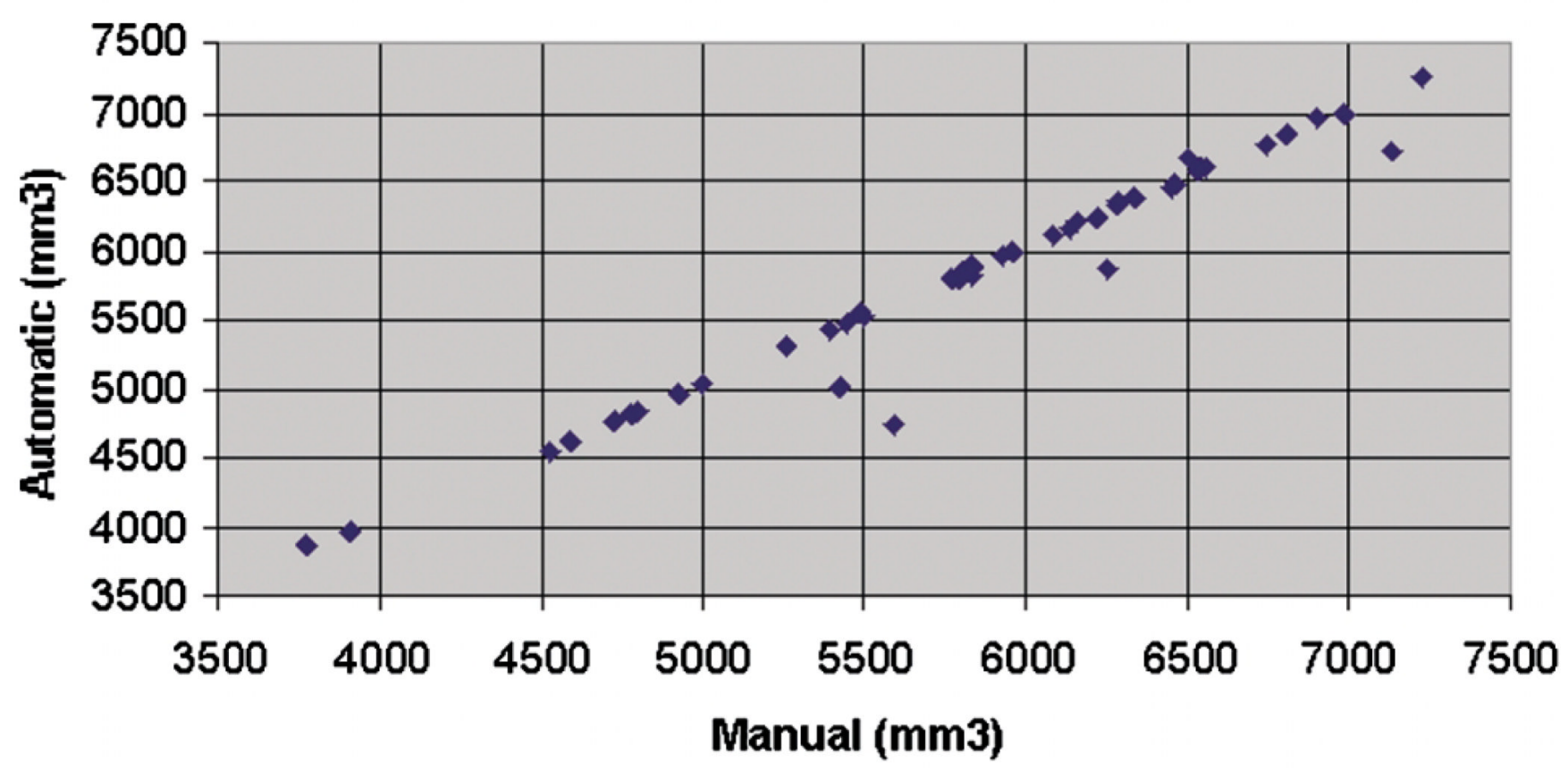

Fig. 4.

Correlation between manual and model-based 3D segmentation of the left and right amygdalahippocampal complex for 21 subjects. The correlation coefficient for the volumetric results is 0.98 . 
Volume Asymmetry: L-R index

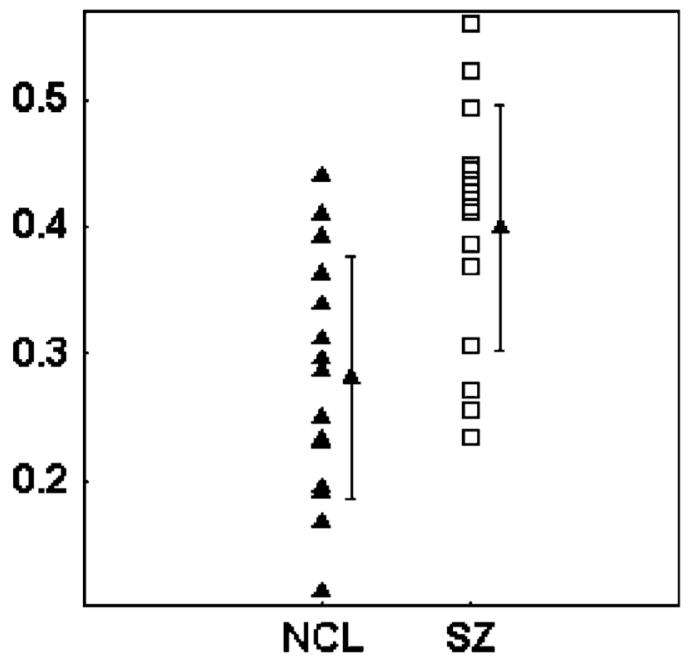

Shape Asymmetry: MSD

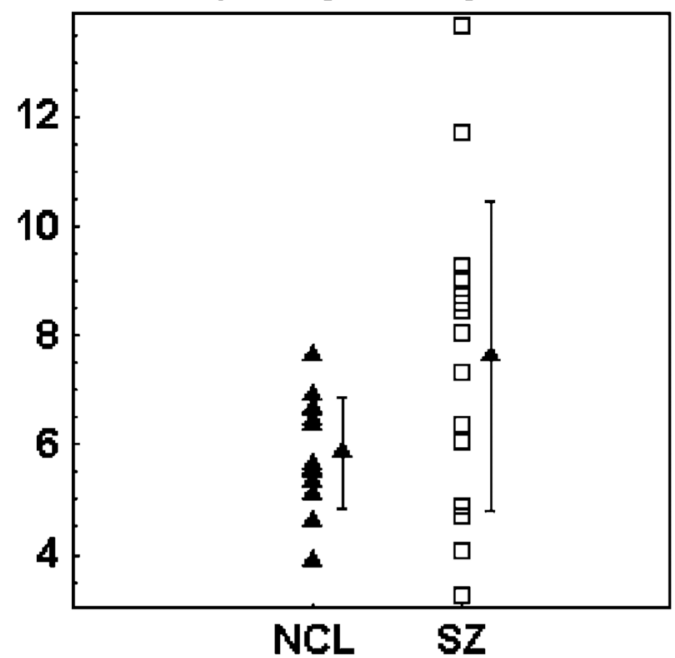

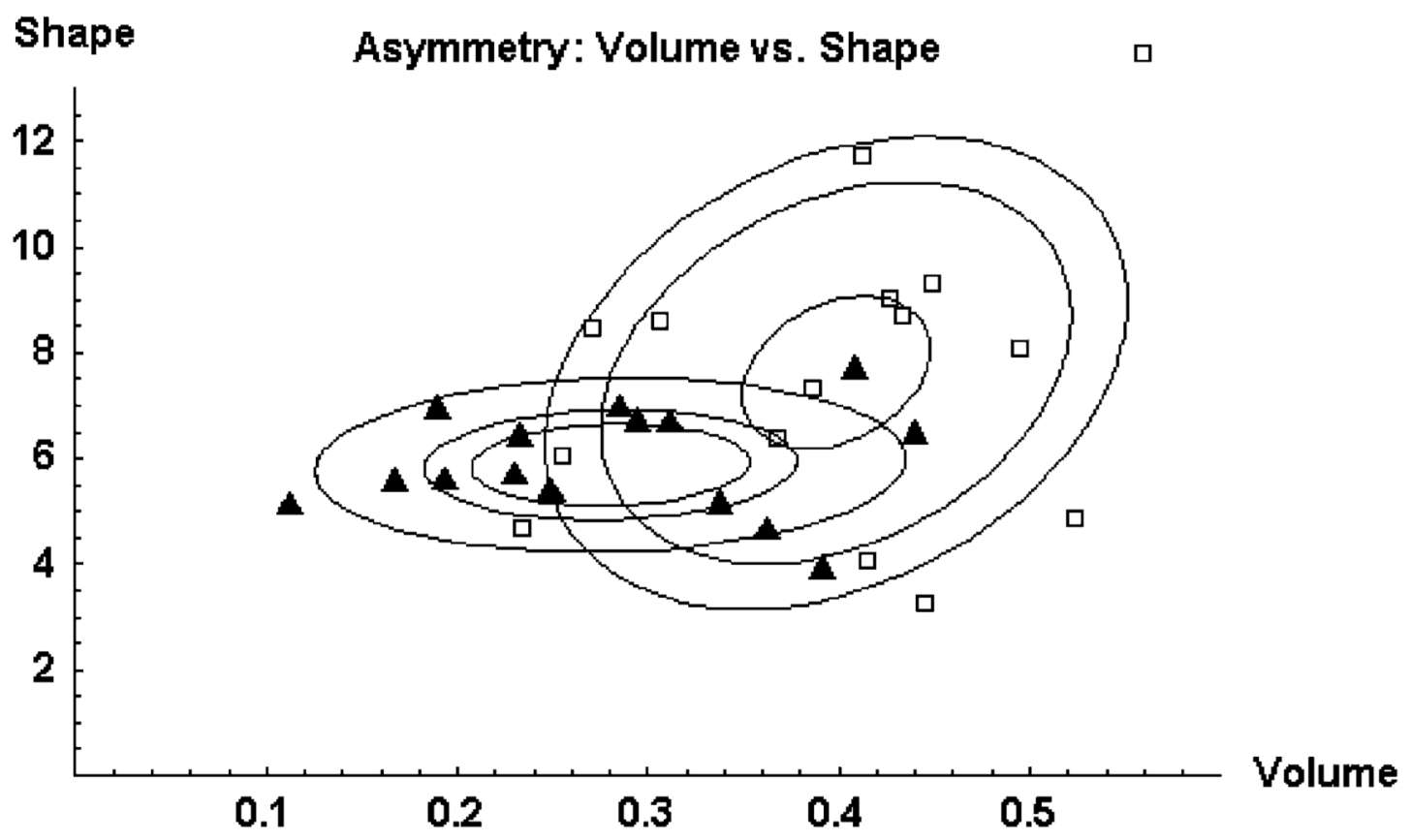

Fig. 5.

Statistics of L/R volume index (upper left panel), L/R shape index (upper right panel), and a combined two-dimensional feature space (bottom panel) with volume index (horizontal axis ) and shape index (vertical axis ). The ellipsoids represent the quantiles of the two-dimensional distributions for controls (black triangles) and for the schizophrenics (open squares). The twodimensional plot demonstrates the improved group discrimination obtained by combining the two features. 

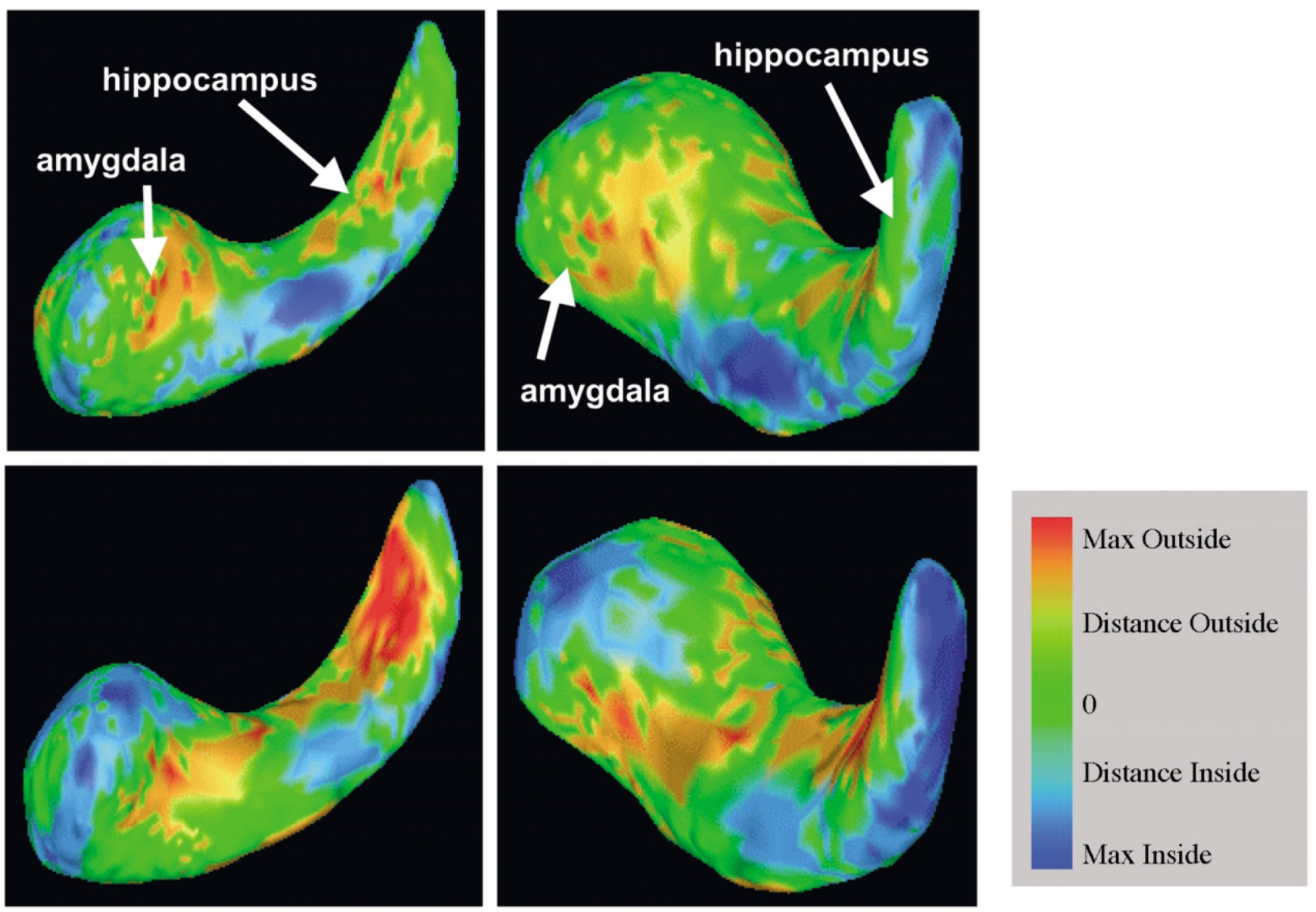

Fig. 6.

A graphical visualization is presented for the left/right asymmetry of the amygdalahippocampal complex for healthy controls (top row) and patients with schizophrenia (bottom row). The left and right columns show sagittal and posterior-anterior viewing directions (i.e., viewing from the tail of the hippocampus). The color figures display group averages of individual pairwise left/right difference calculations. These averages are obtained by mirroring the right shapes to the left, and then overlaying the individual pairs of the amygdala-

hippocampus surfaces. Shapes were uniformly scaled for normalized volumes as described in the text. Signed local surface distances are mapped onto the reference shape as color, ranging from dark blue (maximum inside) to red (maximum outside). At dark blue locations, the right object surface is inside with respect to the left object, whereas at locations of red regions, the right object is outside. Green signifies perfect overlap. The color range is adjusted to plus/ minus $1.4 \mathrm{~mm}$ for maximum inside and outside. A comparison between the healthy control group (top row) and schizophrenic group (bottom row) illustrates that the major local regions responsible for the shape difference are the tail and portions of the amygdala body. The maximum surface distances for healthy controls and for schizophrenics were 1.3 and $2.1 \mathrm{~mm}$, respectively. Please note that the colors do not indicate significance by $P$-value but instead they indicate the magnitude of inside-outside distance between group means. The figures are intended to give preliminary insight into the nature and localization of the quantitative shape asymmetry as described in this article. 


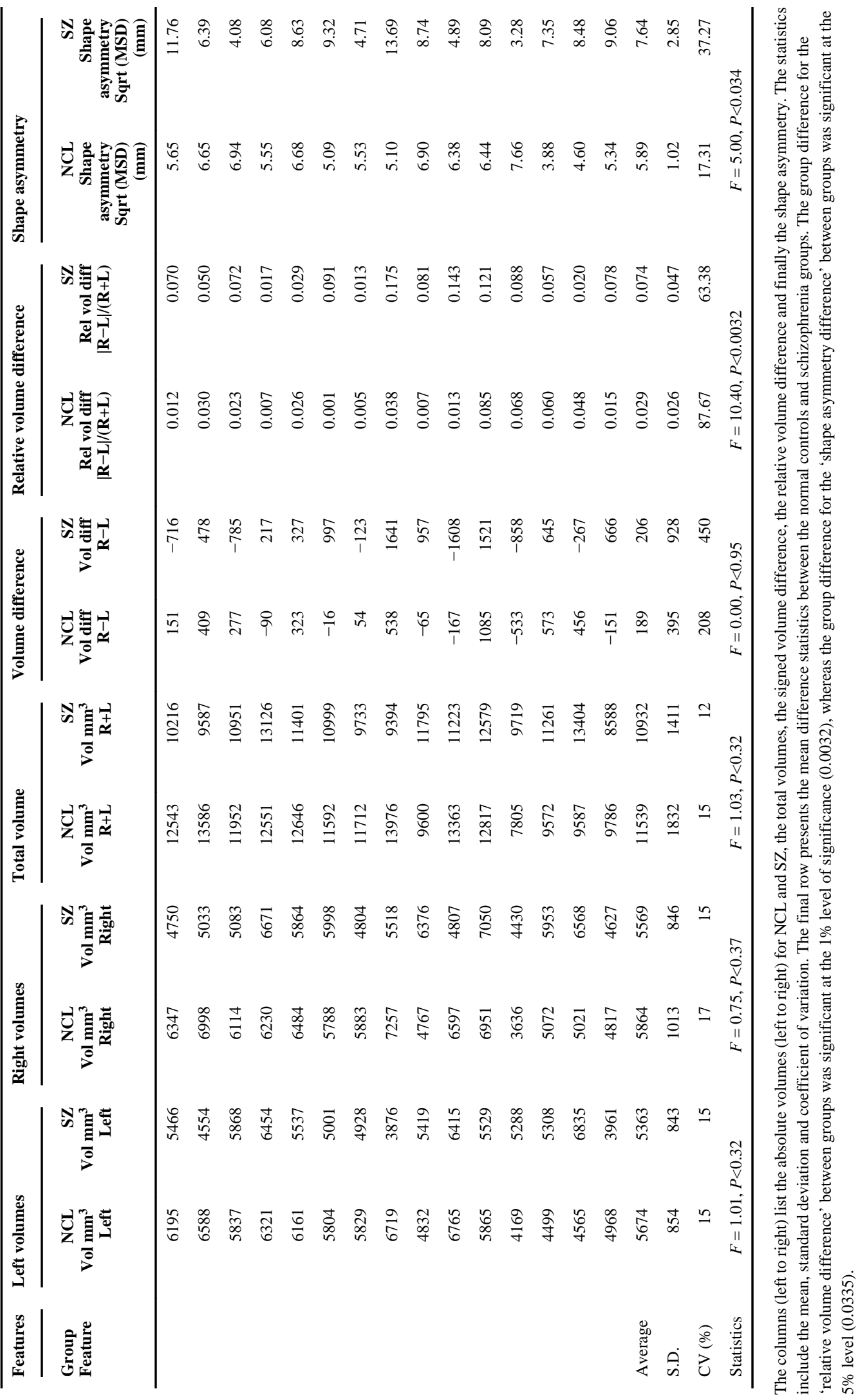




\section{Table 2}

Statistics of left/right shape difference analysis of amygdala-hippocampal complex using the surface representation

\begin{tabular}{llll}
\hline & Volume & Shape & Volume and shape \\
\cline { 2 - 3 } & Rel. L-R diff & MSD surfaces & \\
\hline Student $t$-test $P$ & $F=10.4, P<0.0032$ & $F=5.0, P<0.034$ & $F=11.19, P<0.0024$ \\
Classification & $70 \%$ & $73 \%$ & $87 \%(\mathrm{SVM})$ \\
\hline
\end{tabular}

Classification is poor and not significantly better than a guess for volume and shape only, whereas the combined analysis shows a very good classification rate despite the small numbers of 15 controls and 15 schizophrenics. Classification of the two-dimensional features was carried out with a support-vector-machine technique and leave-one-out. 